\title{
The Progression of Dynamic Reconfigurable Intrusion Identification Wireless Sensor Network
}

\author{
Hathal Salamah A. Alwageed \\ Department of Computer Engineering and Network \\ Aljouf University, Saudi Arabia
}

\begin{abstract}
WSN's are anticipated turn out to be an imperative implementation for an assortment of safety measures and for miscellaneous monitoring applications. We have witnessed and detailed the growth and expansion of a WSN's with having manifold formation of intrusion recognition and categorization. Initially, reasonably trouble-free nodes by means of indispensable sensing devices, a low computational power microcontroller and faculties of wireless transmission are brought into play. The primary stage nodes are exercised as preface intrusion recognizers/detectors. The subsequent/following sensor node is constructed in the order of a towering performance/act FPGA dealing out an assortment of cameras. Such nodes are able to be vigorously built up to carry out diverse forms of scrutiny and visual data processing to bear out and authenticate the existence of every intruder/burglar/imposter in the prospect and to endow with further facts in relation to the intruder. Specifically, the network is capable of lending a hand to an individual operator to substantiate the system's assessment weather an identified or discovered intruder is an impending threat.
\end{abstract}

\section{Keywords}

Intrusion Identification, WSN

\section{INTRODUCTION}

In an un-organized and untried setting, for a system to perform independently or partially dependent, tolerable and ample sensing qualifications are the main concerns. The sensing devices/hardware's networks are one of the up-andcoming promising expertises for bestowing such systems with awareness or acquaintance about position and circumstances in an outsized section of the atmosphere. An immeasurable quantity of sensed data that ought to be get hold of and practiced in real time entails equally embedded hardware or incorporated hardware processing algorithms and smart and quick selection approaches of data sequentially to put a stop to informational diffusion [1]. WSN's are being brought into play for a range of state or situation based monitoring/surveillance applications for example atmosphere or, ecological monitoring [2], Applications for monitoring machineries and aerospace and for protection and supervision [3][4]. More than 10 hundreds of nodes are required for such kind of applications. Each and every node is supposed to be operational with an assortment of sensors like temperature, immediacy/nearness, acoustic, vibration to keep an eye on the state and necessities of an atmosphere. For applications similar to protection and supervision/monitoring, Image sensors are obligatory that involves more comprehensive scrutiny of the atmosphere/settings. Dealing with flow of massive information within the network of nodes is an essential defy here. Broadcasting unprocessed data from each node to the privileged parents and lastly to the targeted node for long duration to take up the bandwidth. This sequentially trims down the system competence by exposure any imperative affair following a long time or by losing any imperative occasion at some stage in the preceding values broadcasting. The entire unprocessed/raw data transmitted/broadcasted from the concern sensor nodes would not be constructive constantly, nearly all of the time throwing away the required obligatory energy for communication and deluging the targeted node with an ineffective data.

To handle the such vast flow of information and stay away from ineffective communication amongst the sensor nodes, integrated field programmable gate arrays with a number of nodes to practice the information nearby and pass it to the privileged parents and in conclusion to the target. Likewise, visual measurement of a state by an individual is at a standstill well thought-out to be the crucial aspect in captivating vital verdicts. The unrefined data conveyed transversely the network would construct the individual scrutiny extremely complicated in multifaceted set-ups. Such FPGA based nodes can process the established data acknowledged from further nodes and process the concern images to investigate the circumstances. In case of infringement they send out the outcomes of the investigated image to the individual operator to verify whether the acknowledged intruder is an impending threat.

Discussion has been made here to identify and make a way out, that how can the functionality/performance of sensing nodes network can be progressed by implanting a towering rank of self-sufficiency along with aptitude in to FPGA-based nodes of multi-sensor networks.

\section{PROPOSED METHOD}

An outsized number of nodes will be put into practices in applications archetypal or standard WSN to process the entire data from an assortment of sensors and also competently handles the power simultaneously to prolong the life of battery [6]. In recent years, in support of unusual applications, a range of structural designs have been projected. [5] [6][7][8]. A lot of work has been carried out on power management in the WSN's whereas some of research has been done on overall designing of system. Two diverse levels of nodes have been put into practice here. The initial/first level nodes are comparatively straightforward through vital sensing devices like immediacy/nearness, acoustic/auditory, vibration, wireless transmission and magnetic sensors qualifications. In the sheltered region they keep an eye on circumstances and operate as opening detectors of likely infringements/intrusion. It puts away incredibly low power and is put together in the order of a low computational power micro-controller. The sensor nodes of the 2 nd or subsequent level are put together around an advanced performance FPGA dealing with an array of cameras. They act upon extremely superior data processing to authenticate or decline the 
intrusion prior to any notification to an individual. Each and every camera is triggered subsequent to the analogous sensor nodes of level 1 st to get hold of data that may possibly point out the intruder existence, and put on the air the notification or alert to the FPGA based sensor nodes of subsequent level in WSN.

\subsection{Sensor Nodes of $1^{\text {st }} /$ Primary Level}

These nodes by and large exercise especially undemanding intrusion recognition approach. Such as, analog signals by means of binary signal thresholding and restricted aptitudes of signal processing with having assumptions to be incessantly on the go. In support of detaining a predefined mishmash of sensor signals from the scrutinized region, a message is passed on in wireless network to the subsequent level nodes to carry out extremely meticulous investigation on the incident. Send out short messages and squat computations make them to put away low power. There is no as such first class research or progression or further development has been made in this particular area and due to which these nodes are understood to be static.

\subsection{Sensor Nodes of $2^{\text {nd }} /$ Subsequent Level}

These nodes are built-in with a field programmable gate array in view of that fact to execute the image processing and multifaceted data in real time. The positioned cameras are usually stationary apart from the infrequent environment image updates. The nodes of this level s are set in motion later than taken delivery of a notification from the node of first level and on commencement detain a number of frames of an image of that fastidious position. These also act upon on intricate operation of image processing to substantiate socalled intrusions and to sort out their temperament. According to the application, to identify an impending treacherous invasion, these nodes wirelessly put out the investigated outcomes or by means of an intruder picture. In particular, FPGA modules/unit is able to be utilized to animatedly reconstructed or put together for unusual odd jobs or diverse applications. The burning up of the Peak energy may possibly be sky-scraping; nevertheless the typical obligation of power is abridged to the height endowing with extended processing time.

\subsection{Realization or Execution of Nodes}

An economical micro-controller, wireless communication chip and other basic sensors have been integrated in primary level of sensor nodes. Power has been bestowed to such nodes in the form of batteries. In favor of the low-power wireless networks, a standard and an acknowledged communication protocol is brought into play. As far as the subsequent level is concern so in that scenario the node slots in the wireless module/units of primary level node, a top rated performance FPGA device with a CPLD unit and an outer memory, CCD cameras and camera interfaces. The supply of battery power to this level node is anticipated, however at this time an adapter of power supply is brought into play. In the primary mock-up/archetype, chosen sections of saleable offered expansion boards have been exercised. At this time, devoted platforms have been assembled.

\section{RECOGNITION/IDENTIFICATION AND CATEGORIZATION OF AN INTRUSION}

An investigation from the sight visuals lends a hand to put together unyielding conclusions and in addition instantly bestows further information to an individual about an event. Diverse systems brought into play for supervision/spying, monitoring applications have been conferred in [3][4][7]. FPGA's and Cameras both has been utilized all together to act upon the obtained images, investigate it in different manners and lastly propel their consequences for further processing to an individual, in view of the fact that if the intrusion has been occurred. With the intention to that, there is call for the cameras not to make efforts in an endlessly manner, which contrary may perhaps upshot in an extreme power expenditure. Simultaneously, with in the environment, the system won't fail to notice any intruder. A variety of algorithms concerning image processing have been built-in to carry out unusual or special tasks. There is an image preprocessing operations that has been put into operation in FPGA and which is being used by such algorithms. Usually, [3] we do have three predictable methods for the pulling out or removal of stirring objects from a video stream. They are sequential/temporal differencing, optical/visual flow and background subtraction. The temporal differencing is extremely flexible or supple to self-motivated and active settings; however don't have such aptitude to execute fit in pulling out all pertinent characteristic pixels. An optical flow computation technique is acknowledged to be incredibly multifaceted and irrelevant to concurrent applications other than dedicated hardware. Background subtraction is exceptionally responsive to self-motivated surroundings other than endow with an absolute facet data. Here background subtraction has been brought into play by means of added image processing algorithms to pay damages for the instabilities of observable state of affairs. After pulling out the objects by weigh against the background images, it is compulsory to frequently keep posted the background images to acquire further faultless outcomes. The background images of the environment is being kept posted or updated by the 2nd level nodes, while they are unoccupied and/or in case where they don't identify any fresh targets in the scenes. In the company of up-to-the-minute objects/targets, it has the ability to propel the information regarding the object to an individual (operator), and just in case it is not well thought-out treacherous, it will onwards be comprised in the subsequent update. This may perhaps also fluctuate as per application having mandatory unusual background updates requirements.

\subsection{Identification of Fixed or Motionless Intrusion}

As far as standard applications of WSN's are concern, so nodes in an enormous number will be brought into play and the assessment of data quantity for an individual can be very hefty. Such as, when in a scenario of forest with the settings of having few hundred or more nodes are employed, it is over and over again tricky for an individual always to recognize the intruders as roughly all images seem to be awfully related. When a cautious note is usual from the primary level nodes demonstrating the company of any intruder, the intruder image are pulled out by putting side by side the contemporary images to the environment image. Methods of mathematical morphology and threshold values have been utilized just for the reason to ignore slight instabilities in the background. Additional investigation of the intruder's is feasible to be carried out by making use of moments and moment invariants practices to consent an extensive categorization in preferred applications. Further scrutiny regarding intruder can be furnished and carried out by putting side by side the moments of the identical/similar intruder in an assortment of image 
frames.

\subsection{Identification of active and self- motivated Intrusion}

To investigate the intruder vitality, the deviation of the shape of intruder are removed or pulled out from a succession of images. Which in point of fact point towards the movement of intruder and the upshots can be later brought into play to sort out the type and mobility of the intruder.

\subsection{Identification of Interest Points}

We may perhaps comprise an initiative about the potential intruders in a number of the applications, and in such scenario the system will be able to make out whether the identified intruder is that one of the intruders within the data base. Such is feasible to be accomplished by means of identifying a number of interest points in the image of the intruder. After doing so, the information can be sent out to the superior nodes inside the conclusion succession. Later on it (interest points) can be obtained by the target system by means of an images database identified intruders. This technique in point of fact trims down the quantity of data broadcasted within the wireless network, in so doing diminishing the utilization of power. The broadcasted interests point can be harmonized [10] with the identified intruder's database to make a decision about the intruder. Such scheme/approach constructs the system completely self-governing nevertheless all along with the constraints such that it will not be able to be functional to the applications of not having any knowledge about the potential intruders.

\section{RESULTS BROADCASTING}

later than an assortment of investigation of the intruder, the 2nd level nodes is able to either broadcast or send out the area of interest of the image or statistical values to the superior level in the decision/conclusion chain and in conclusion to an individual for an ultimate result. For such applications when enormous quantities of cameras are brought into play, each and every node may possibly sends out stacks of images within the network which may perhaps turn out to be the reason for a tailback inside the network. For such images its area of interest can be packed together nearby prior to the broadcasting/transmission to lessen the data quantity to be sent out. A number of researches have been carried out to evaluate a range of compression prototypes to be brought into play in inside the WSN's applications for a well-organized and productive management of power [6][11].

Such algorithms is also feasible to be intended to acclimatize convinced parameters like ratio of compression, resolution of image based on the synchronized condition [6][12][13]. Advanced encryption standard (AES128) has been utilized to encrypt the fragments of image prior to the transmission to endow an improved safety measures well thought out to be an imperative prerequisite for loads of applications.

\section{CONCLUSION}

A structural design based on a multifold level of nodes can be brought into play for the identification and categorization an intrusion within the WSN's. In this article a variety of processing algorithms incorporated in FPGA and visual measurements has been outlined. The 2 nd level nodes based on FPGA are quite competent and be able to be energetically put together for diverse and unusual range of applications and responsibilities as well, subsequent to the exploitation of the nodes bringing into play the qualifications and aptitudes of an online re-configuration.

\section{REFERENCES}

[1] "Obraczka et al", "Managing the Information Flow in Visual Sensor Networks,"' Proc. WPMC 2002: The Fifth International Symposium on Wireless Personal Multimedia Communication, October 27--30, 2002 , Honolulu, HI

[2] "Estrin", "Sensor network research: Emerging challenges for architecture, systems, and languages," in Proceedings of the 10th International Conference on Architectural Support for Programming Languages and Operating Systems (ASPLOS-X), ser. ACM SIGPLAN notices, C. Norris and J. J. B. Fenwick, Eds., vol. 37, 10. New York: ACM Press, October 5-9 2002, pp. 1-4.

[3] "Collins et al", "A System for Video Surveillance and Monitoring," in Proceedings of the American Nuclear Society (ANS) Eighth International Topical Meeting on Robotics and Remote Systems, April, 1999.

[4] "Collins et al", "Algorithms for cooperative multisensor surveillance," Proceedings of the IEEE, Vol. 89, No. 10, October, 2001, pp. 1456 - 1477.

[5] "J. Bellis et al", "Development of Field Programmable Modular Wireless Sensor Network Nodes for Ambient Systems", Computer Communications - Accepted for Special Issue on Wireless Sensor Networks, to appear in 2005.

[6] "John Lach et al", "Power-Efficient Adaptable Wireless Sensor Network",. Military and Aerospace Programmable Logic Devices International Conference, 2003.

[7] "Meffert B et al ", Winkler F: "Monitoring traffic by optical sensors". Second International Conference on Intelligent Computing and Information Systems Proceedings, pp. 9-14.

[8] http://mediax.stanford.edu/projects/cvsn.html

[9] "M.S. Islam et al", "Towards invariant interest point detection of an object," Proc. $13^{\text {th }}$ International Conference in Central Europe on Computer Graphics, Visualization and Computer Vision, pp. 101-104, Czech Republic, 2005.

[10] "M.S. Islam et al", "Matching interest points of an object," IEEE International Conference on Image Processing, Genova, Italy, September 11 -14, 2005 (accepted).

[11] "H. Wu et al", "Energy Efficient Distributed JPEG2000 Image Compression in Multihop Wireless Networks," in Proceedings of the 4th workshop on Applications and Services in Wireless Networks (ASWN), Boston, Massachusetts, 2004

[12] "N. Taylor et al",. "Adaptive image compression for enabling mobile multimedia communication", in Proceedings of IEEE International Conference on Communications, 2001.

[13] "N. Taylor et al", "Energy/latency/image quality tradeoffs in enabling mobile multimedia communication", in E. D. Re, editor, Software Radio - Technologies and Services, pages 55-66. Springer Verlag, 2001. 